\title{
Electronic Metal-Support Interactions at the Catalytic Interfaces of CuO Nanowires Decorated with Pt Nanoparticles for Methanol Oxidation and CO Sensing
}

Zakaria Ziadi, ${ }^{\dagger},{ }^{\dagger}$ Alexander J. Porkovich, ${ }^{\dagger}$ Pawan Kumar, ${ }^{\dagger}$ Abheek Datta, ${ }^{\dagger}$ Eric Danielson, ${ }^{\dagger}$ Vidyadhar Singh, ${ }^{\dagger}+$ Toshio Sasaki, ${ }^{\S}$ and Mukhles Sowwan ${ }^{\dagger}$

+ Nanoparticles by Design Unit, Okinawa Institute of Science and Technology (OIST) Graduate University, 1919-1 Tancha, OnnaSon, Okinawa 904-0495, Japan

‡ Department of Physics, Jai Prakash University, Chapra-841301, Bihar, India

$\S$ Imaging Section, Okinawa Institute of Science and Technology (OIST) Graduate University, 1919-1 Tancha, Onna-Son, Okinawa 904-0495, Japan

*E-mail: zakaria.ziadi@oist.jp

Section S1. CuO Nanowire (NW) Based Gas Sensing Device Fabrication

Figure S1. Schematic of Lithographic Process

Figure S2. Pt nanoparticle (NP) decoration process of a CuO NW based gas sensing device

Section S2. Scanning Transmission Electron Microscopy (STEM) of Pt Nanoparticles (NPs) on CuO NWs

Figure S3. STEM image of two Pt NPs on a CuO NW shown in Figure $1 \mathrm{~b}$ with a different focus

Figure S4. A full HAADF-STEM image of Figure 1c with analysis

Section S3. High Resolution X-ray Photoelectron Spectroscopy (XPS) Supplement

Figure S5. XPS spectrum of the $\mathrm{Cu} 3 p$ doublet of pristine $\mathrm{CuO}$ nanowires

Figure S6. XPS spectra of Pt $4 f, C 1 s, O$ 1s and Si $2 p$ for Pt NPs on an Si substrate

Figure S7. XPS spectra of $\mathrm{C} 1 \mathrm{~s}$ for pristine CuO NWs, as-deposited Pt NPs on CuO NWs and Pt NPs on CuO NWs annealed at 200 and $250{ }^{\circ} \mathrm{C}$

Figure S8. XPS spectra of the $\mathrm{Cu} 2 p$ doublets for pristine $\mathrm{CuO}$ NWs, as-deposited Pt NPs on CuO NWs and Pt NPs on CuO NWs annealed to temperatures of 200 and $250{ }^{\circ} \mathrm{C}$

Figure 59. XPS spectra of $\mathrm{C} 1 \mathrm{~s}$ for pristine CuO NWs, as-deposited Pt NPs on CuO NWs and Pt NPs on CuO NWs annealed to temperatures of 200 and $250{ }^{\circ} \mathrm{C}$

Section S4. PeakForce Kelvin Probe Force Microscopy (PF-KPFM) Supplement

Figure S10. Topographical images corresponding to the surface potential images seen in Figure 4

Figure S11. Surface potential distribution profiles corresponding to the surface potential images of Figure 4

Section S5. Conductometric Measurements of CuO NW Based Devices and CO Gas Sensing Measuremtns

Figure S12. Current through pristine $\mathrm{CuO} \mathrm{NW}$ based devices at 200 and $250{ }^{\circ} \mathrm{C}$

Figure S13. Current through Pt NP decorated CuO NW bases devices at 200 and $250^{\circ} \mathrm{C}$

Figure S14. Relative resistance response to $\mathrm{CO}$ gas of a pristine and $\mathrm{Pt}$ decorated $\mathrm{CuO}$ NW based devices at $200^{\circ} \mathrm{C}$

Section S6. References 


\section{Supporting Information}

\section{Section S1. CuO Nanowire (NW) Based Gas Sensing Device Fabrication}

a)

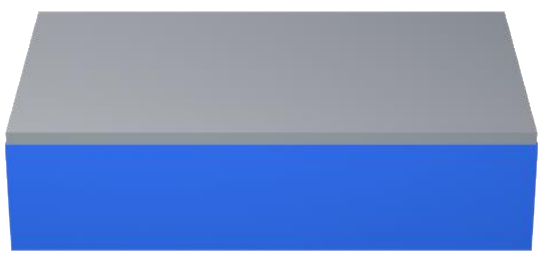

c)

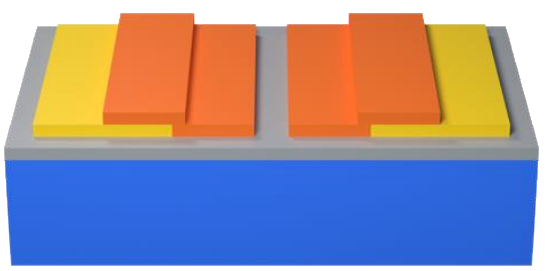

b)

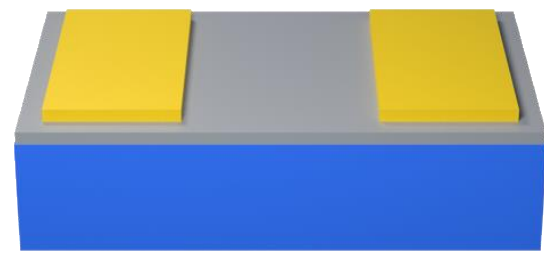

d)

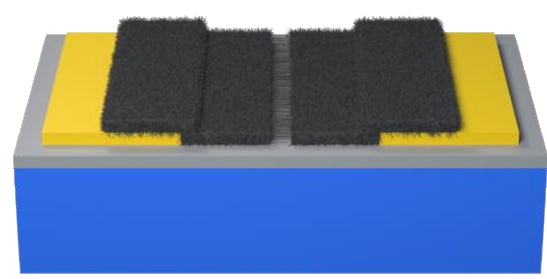

$\mathrm{Ti} / \mathrm{Cu}$

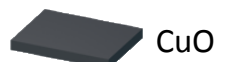

CuO NWs

Figure S1. Diagram of the fabrication process of CuO NW based gas sensing devices. a) a $500 \mu \mathrm{m}$ of (100) Si wafer with a thermally grown $300 \mathrm{~nm} \mathrm{SiO} 2$ insulating layer is used as substrate. b) A liftoff lithography process using a positive photoresist (Shipley S1830) is sued to evaporate an adhesion layer of $5 \mathrm{~nm}$ of Ti followed by a $200 \mathrm{~nm}$ layer of Au as electrical contacts. Using a negative resist (ALLRESIST ARN 44 50), another layer of Ti/Cu (5 nm and $1000 \mathrm{~nm}$, respectively) was deposited over part of the $\mathrm{Au}$ and $\mathrm{SiO}_{2}$ forming the structure shown in c). Finally, CuO NWs growth bridges the initially deposited Cu pads electrodes via thermal oxidation at $350^{\circ} \mathrm{C}$ using a hotplate. 


\section{Supporting Information}

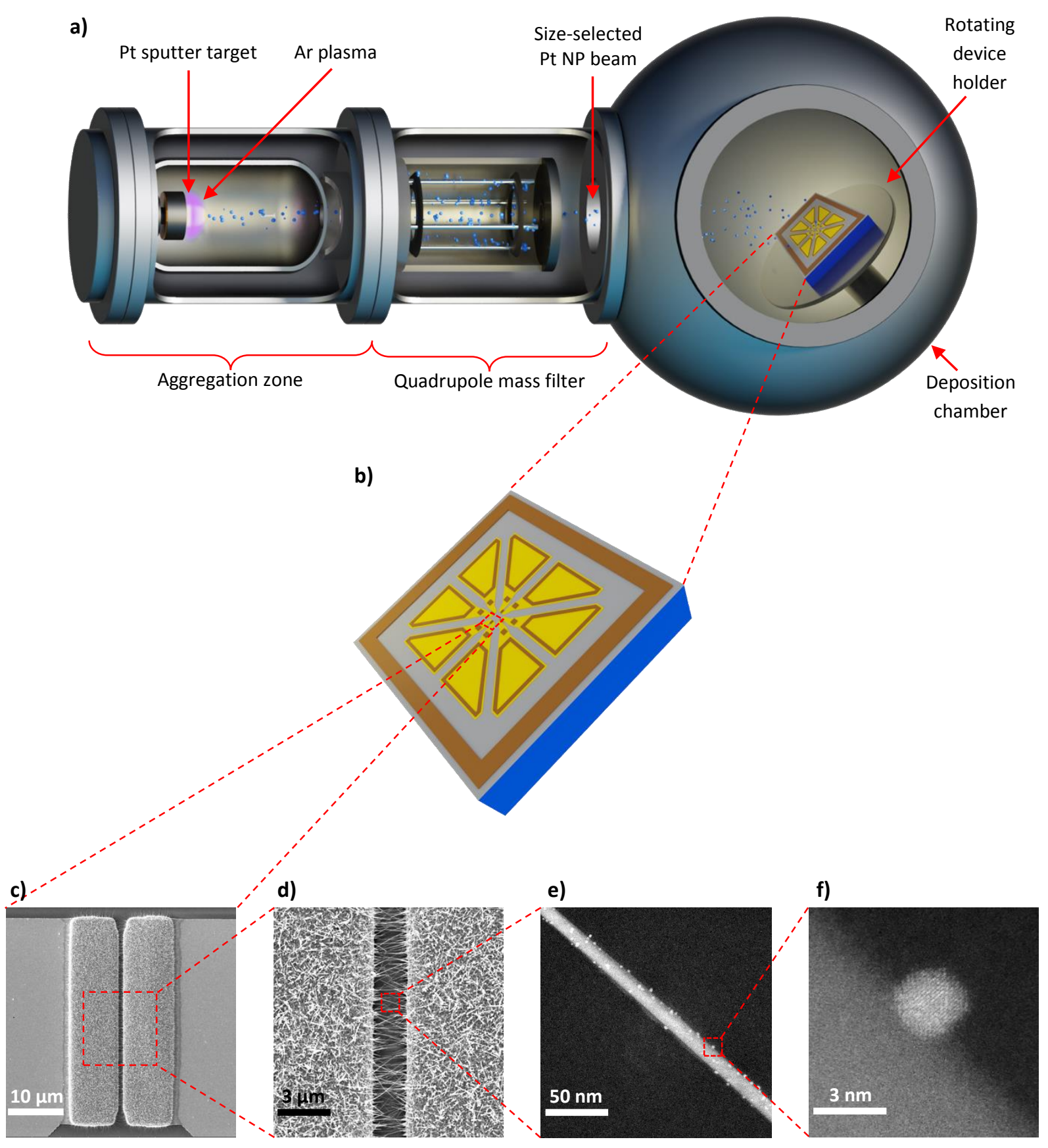

Figure S2. a) Schematic diagram of the magnetron-sputtering system for the gas-phase synthesis and deposition of Pt NPs on a b) CuO NW based device. c) Scanning electron microscopy (SEM) image of one of the four $\mathrm{CuO}$ pads where the nanowires bridge the gap, as shown in the closed-up image in d). A scanning transmission electron microscopy (STEM) image of a CuO NW decorated with Pt NPs is shown in e), while f) shows a high-angle annular dark-field (HAADF) STEM image of a Pt NP on the CuO NW. 


\section{Supporting Information}

Section S2. Scanning Transmission Electron Microscopy (STEM) of Pt Nanoparticles (NPs) on CuO NWs

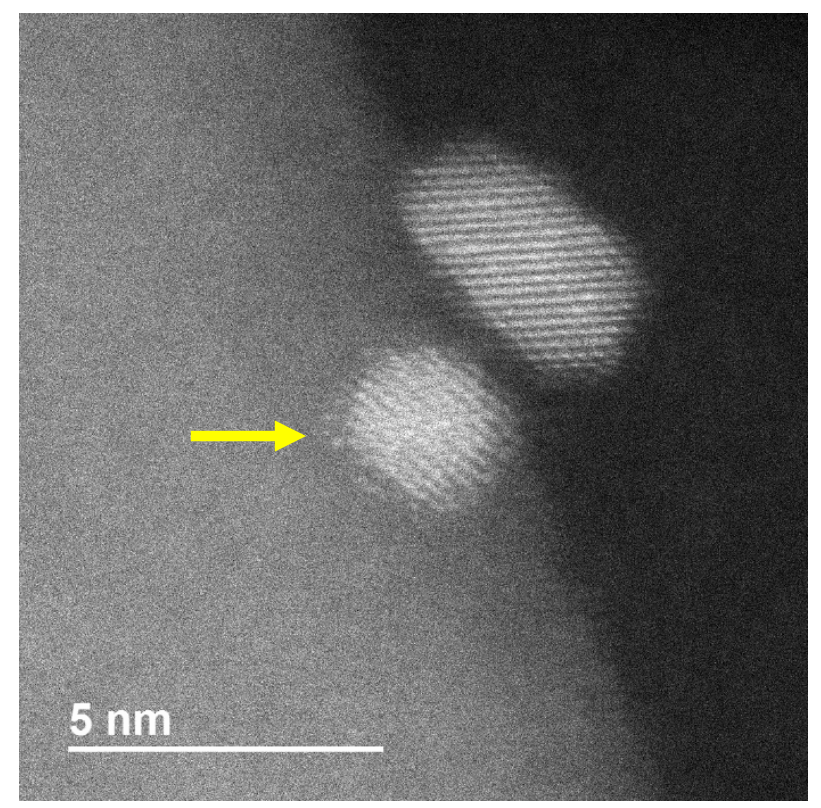

Figure S3. HAADF STEM image of two Pt NPs on a CuO NW shown in Figure 1b with a different focus, showing the crystallinity of the smaller Pt NP (indicated by the yellow arrow).

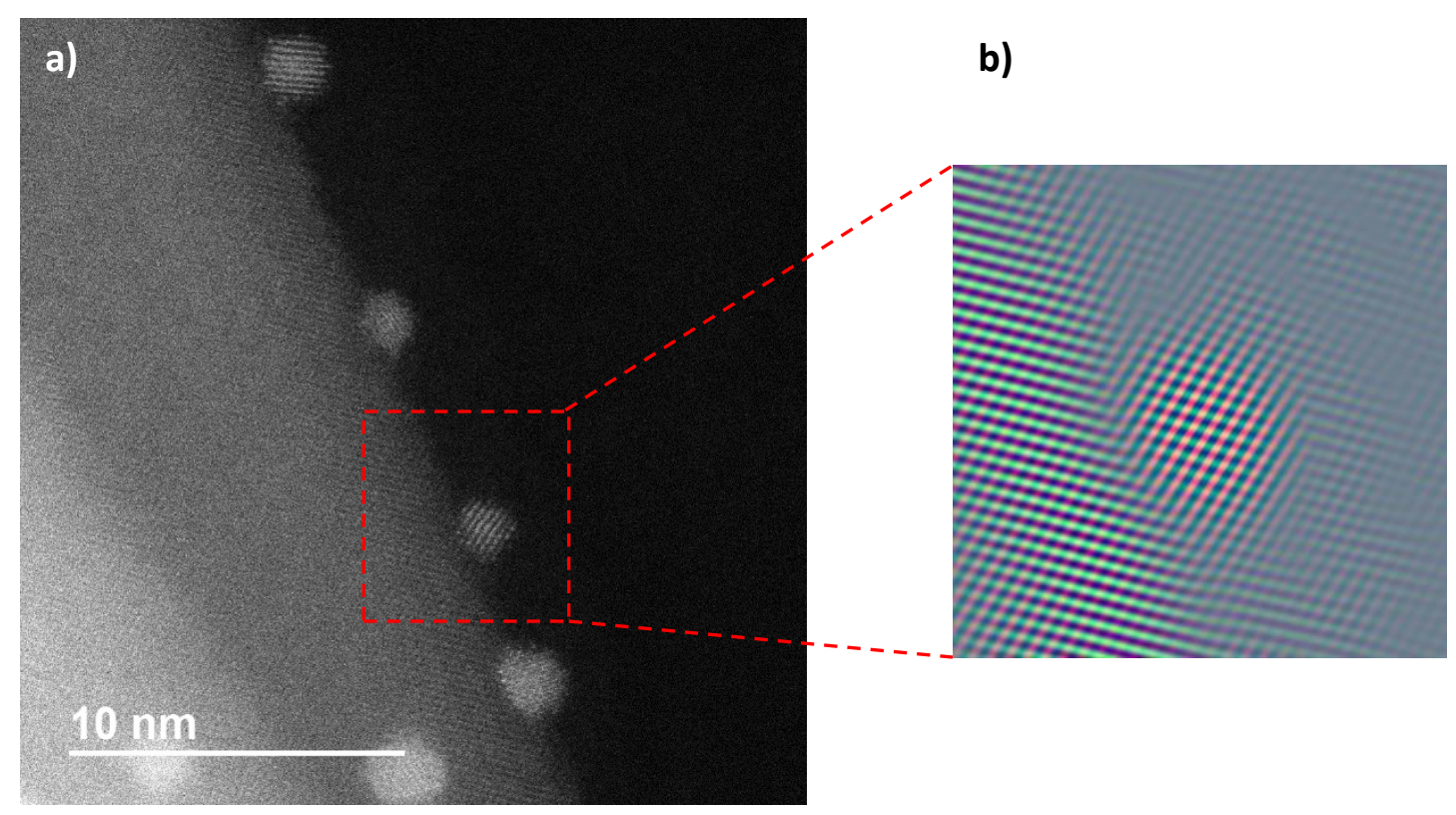

Figure S4. a) A full HAADF-STEM image of Figure 1c with an emphasis on the preferential orientations of the planes of the Pt NP in interest with CuO NW shown in b) as a false color image. The red color depicts the (111) planes of Pt and (200) of the CuO, while the green color is associated with the (200) planes of Pt and (112) for the CuO. 


\section{Supporting Information}

\section{Section S3. High Resolution X-ray Photoelectron Spectroscopy (XPS) Supplement}

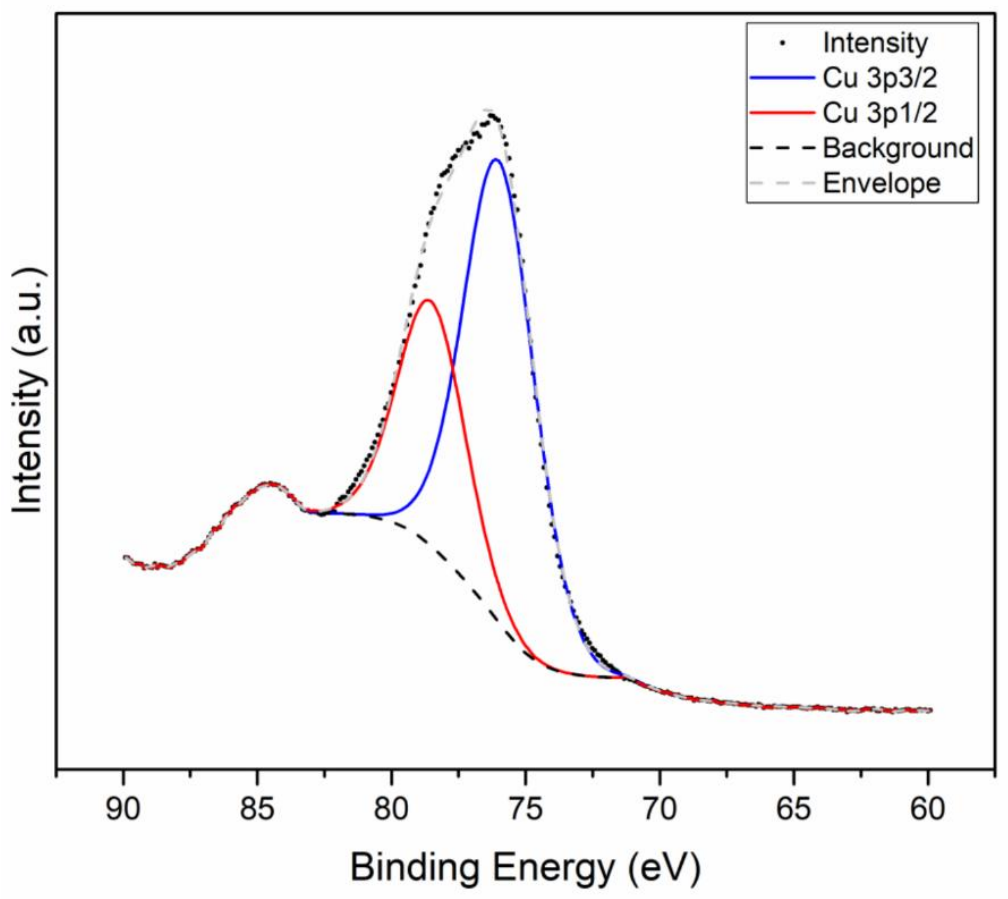

Figure S5. XPS spectrum of the $\mathrm{Cu} 3 p$ doublet of pristine $\mathrm{CuO}$ NWs. The black dots $(\bullet)$ indicate the collected data, dashed black line is the Shirley background, grey dashed line is the convolution of the fitting peaks and the blue (3p3/2) and red (3p1/2) peaks are Gaussian-Lorentzian functions used to best approximate the shape of the $\mathrm{Cu} 3 p$ doublet under oxidised conditions.

Figure S5 shows the fitting of the $\mathrm{Cu} 3 p$ doublet for pristine nanowires. In this case, the shake-up satellite was ignored, and a Shirley background was applied to the doublet only. The 3p1/2 fit was constrained to $50 \%$ of the area of the $3 p 3 / 2$ fit, while both full width at half maximum (FWHM) values were equal. The peaks were found to have a split spin orbit of $2.5 \mathrm{eV}$, with peaks centred at $76 \mathrm{eV}(3 / 2)$ and $78.5 \mathrm{eV}(1 / 2)$ consistent with previous oxidised Cu studies. ${ }^{1}$ 


\section{Supporting Information}
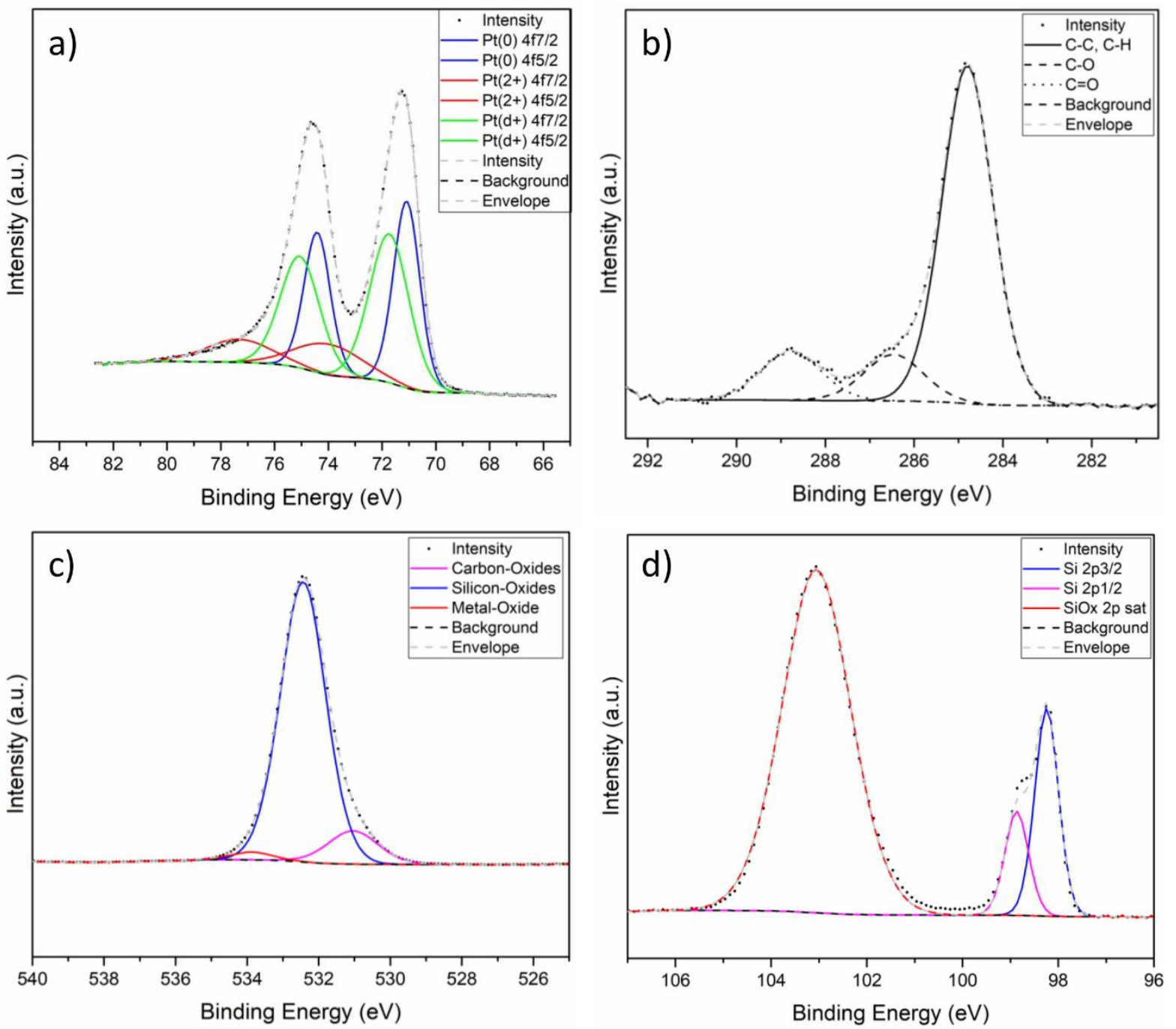

Figure S6. XPS spectra of a) Pt 4f, b) C $1 s$, c) $\mathrm{O} 1 s$ and d) Si $2 p$ for Pt NPs on an Si substrate. The black dots $(\bullet)$ indicate the collected data, the Shirley background is shown by a dashed black line, and the grey dashed line represents the convolution of the fitting peaks in all cases. Blue, red, green and magenta peaks indicate the different deconvolved peaks (including doublets and satellite contributions) and their meaning is described in the legend for each spectrum. In the case of $\mathbf{b}$ ), the solid black line represents the hydrocarbon peak, with the dashed line showing single bond carbon oxides, and the dotted line showing double bond carbon oxides. Charge was corrected by setting the carbon peak to $284.8 \mathrm{eV}$, leaving the metallic Pt 4f7/2 peak at $71.3 \mathrm{eV}$. Metal, carbon and silicon oxides were all confirmed to be present in the $\mathrm{O} 1 \mathrm{~s}$ and $\mathrm{Si} 2 p$ spectra.

Figure S6 shows high resolution XPS spectra of various parts of the Pt NPs on Si substrate spectrum. The Pt $4 f$ doublet has been fit with six Gaussian-Lorentzian functions (Figure S6a), each constrained with a spin orbit split of $3.33 \mathrm{eV}^{2}$ Additionally, each $4 f 5 / 2$ spin is constrained to have $75 \%$ of the area of the corresponding $4 f 7 / 2 \mathrm{spin}$. These represent three doublets related to the metallic $0\left(\mathrm{Pt}^{0}\right), \delta+\left(\mathrm{Pt}^{\delta+}\right) 2+$ and $\left(\mathrm{Pt}^{2+}\right)$ valence state. The resulting fits found the $\mathrm{Pt}^{0}$ doublet in $71.3 \mathrm{eV}$ and $74.6 \mathrm{eV}$, which is consistent 


\section{Supporting Information}

with the literature ${ }^{2}$ for charge correction of $\mathrm{C} 1 \mathrm{~s}$ to $284.8 \mathrm{eV}$ (Figure S6b). The $\mathrm{Pt}^{2+} 4 f 7 / 2$ peak was subsequently found in low concentrations ( $15 \%$ of total Pt species), likely due to adsorbed oxygen on the surface of the nanoparticles, as can be seen in the metal oxide peak in the 01 s peak (Figure S6c). Most of the other oxygen present on the surface is due to the native oxide on the $\mathrm{Si}$ ( $\mathrm{Si} 2 p$ peak as seen in Figure $\mathbf{S 6 d}$ ), while some is due to the formation of carbonic oxides.

Most interestingly is the presence of the $\mathrm{Pt}^{\delta+} 4 f 7 / 2$ peak at $71.7 \mathrm{eV}$. This peak is associated with a depletion of electronic charge on the $\mathrm{Pt} .{ }^{3}$ However, it is not typically related to the formation of a new compounds, but instead, the presence of charge transfer between articles and support. ${ }^{4}$ Overall, the fitted spectrum agrees well with previous reports of $\mathrm{Pt}$ on $\mathrm{SiO}_{2},{ }^{5}$ suggesting that the full surface is covered in a native oxide. 


\section{Supporting Information}
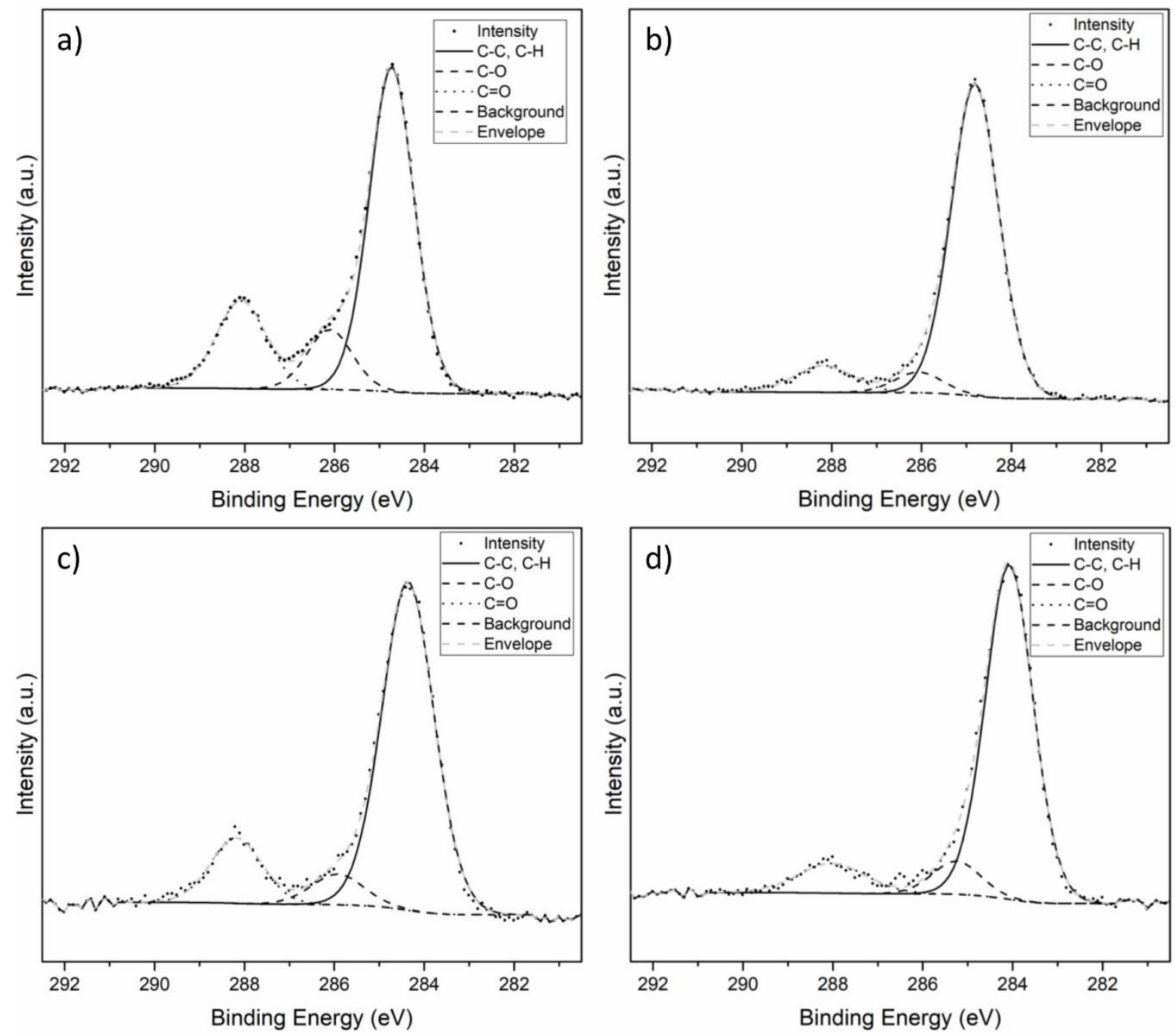

Figure S7. XPS spectra of $\mathrm{C} 1 \mathrm{~s}$ for a) Pristine CuO NWs, b) as-deposited Pt NPs on CuO NWs and Pt NPs on CuO NWs annealed to c) $200{ }^{\circ} \mathrm{C}$ and d) $250{ }^{\circ} \mathrm{C}$. The black dots ( $\left.\bullet\right)$ indicate the collected data, the Shirley background is shown by a dashed black line, and the grey dashed line represents the convolution of the fitting peaks in all cases. The solid black line represents the hydrocarbon peak, with the dashed line showing single bond carbon oxides, and the dotted line showing double bond carbon oxides. Pristine $\mathrm{CuO}$ and as-deposited Pt NPs on CuO NWs are found to be at $284.8 \mathrm{eV}$, while the annealed sample show a continual shift to lower binding energies.

As with the Pt NPs on a Si substrate, all CuO NW measurements were referenced to a carbon C 1s peak of $284.8 \mathrm{eV}$ (Figure S7). Measurements of unannealed pristine and Pt NP decorated CuO NW (Figure S7a and $\mathbf{b}$, respectively) show this peak at $284.8 \mathrm{eV}$. However, the annealed Pt NPs on CuO NWs show an increasing decrease in binding energies (Figure S7c and d, respectively). This is an actual shift of the C $1 \mathrm{~s}$ peak likely due to the changing work function of surface of the nanowire, as this peak has been shown to be sensitive to the work functions of semi-conductors. ${ }^{6}$ The fact that all other chemical peaks appear unmoved is indicative of this as a carbon shift, a fact noted in similar recently reported works involving 


\section{Supporting Information}

annealed Ru decorated $\mathrm{CuO} \mathrm{NWs,}{ }^{7}$ which demonstrated a changing work function with annealing the nanowire-nanoparticle system.
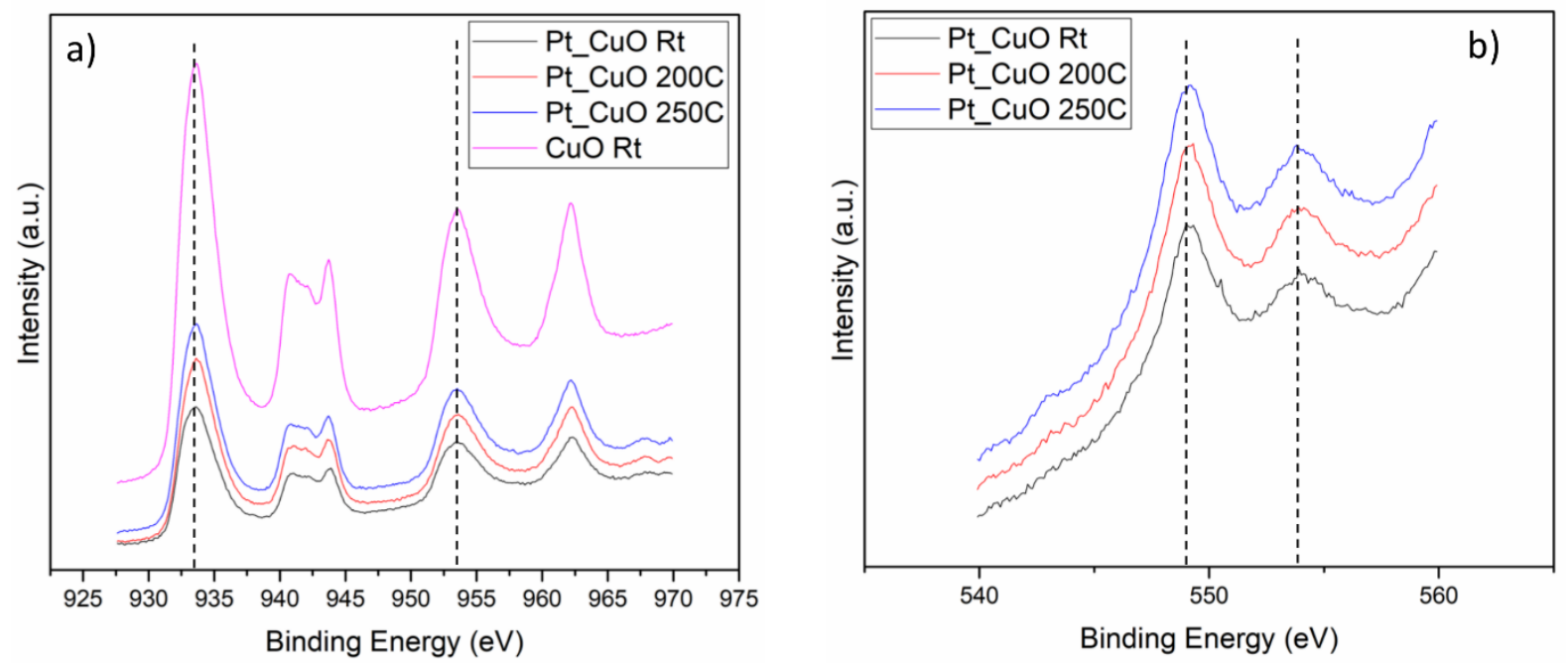

Figure S8. XPS spectra of the a) $\mathrm{Cu} 2 p$ doublets for Pristine CuO NWs, as-deposited Pt NPs on CuO NWs and Pt NPs on CuO NWs annealed to temperatures of 200 and $250{ }^{\circ} \mathrm{C}$. The distance between the $\mathrm{CuO} 2 p$ doublet shows no shift across all samples, indicating no real chemical change of state for the nanowires. The dotted lines have a spin orbit split of $19.9 \mathrm{eV}$, with $\mathrm{Cu} 2 p 3 / 2$ being located at $933.7 \mathrm{eV}$ (Consistent with $\mathrm{CuO}) .{ }^{2}$ This is supported by b) the $\mathrm{Cu} L_{2} M_{4,5} M_{4,5}$ Auger lines for as-deposited Pt NPs on CuO NWs and Pt NPs on CuO NWs annealed to temperatures of 200 and $250^{\circ} \mathrm{C}$. Similarly, the dotted lines indicate the $548 \mathrm{eV}$ position of the $\mathrm{Cu}$ Auger lines (for a monochromatic Al source), ${ }^{2}$ highlighting the samples consistency in chemical state. 


\section{Supporting Information}
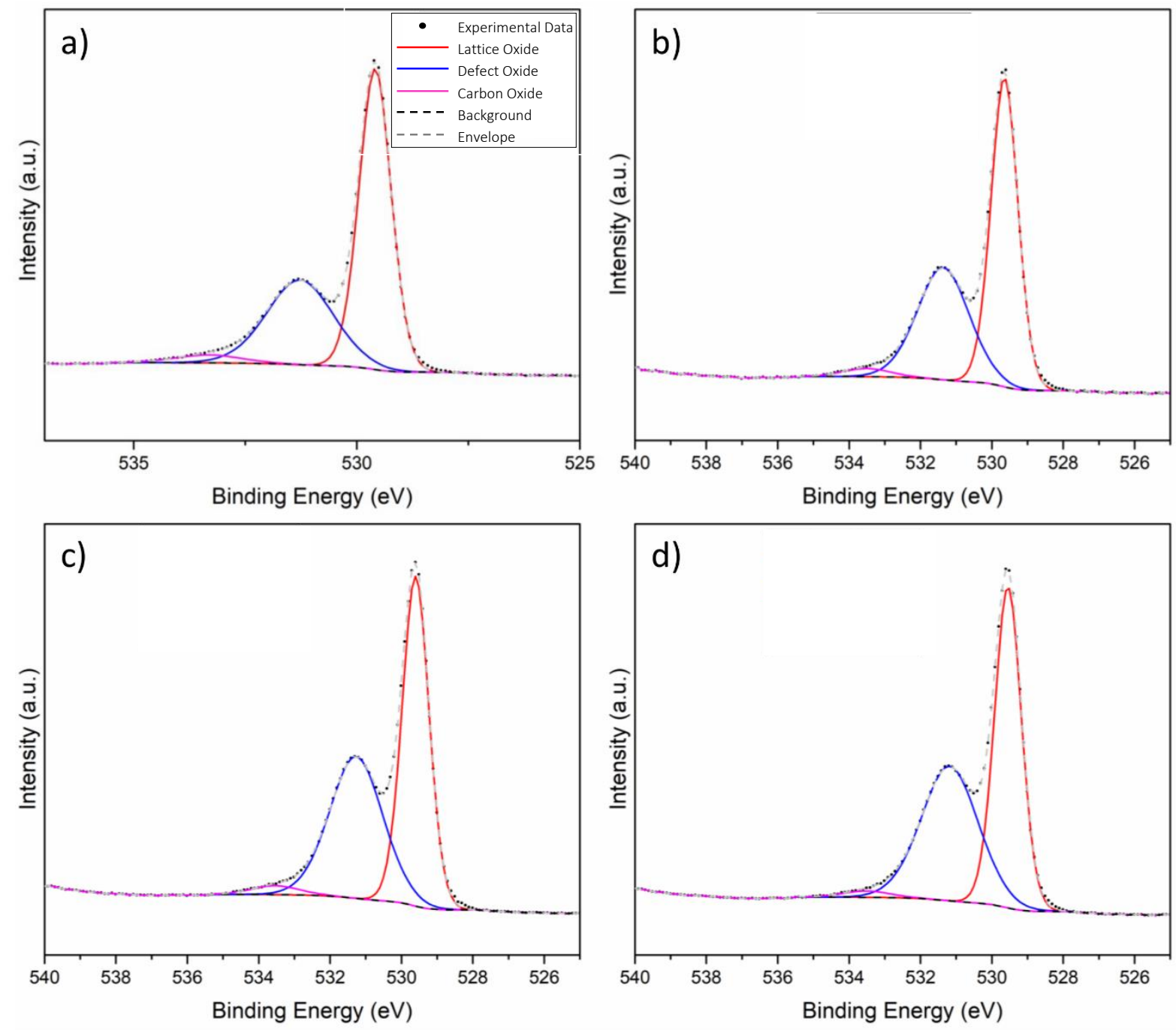

Figure S9. XPS spectra of $\mathrm{C} 1 \mathrm{~s}$ for a) Pristine $\mathrm{CuO}$ nanowires, b) as-deposited Pt NPs on CuO NWs and Pt NPs on CuO NWs annealed to c) $200{ }^{\circ} \mathrm{C}$ and d) $250{ }^{\circ} \mathrm{C}$. The black dots $(\bullet)$ indicate the collected data, the Shirley background is shown by a dashed black line, and the grey dashed line represents the convolution of the fitting peaks in all cases. In all spectra, red lines denote the metal lattice-oxide peak of $\mathrm{CuO}$, blue lines denote an amalgamation of the defect metal oxide of $\mathrm{CuO}$, Pt metal oxide and possible Pt metal charge transfer, and magenta lines denote carbonic oxides.

Figure S9 shows high resolution XPS of the $\mathrm{O}$ 1s peak for pristine CuO NWs (Figure S9a), as-deposited CuO NWs (Figure S9b), and samples of Pt NPs deposited on CuO NWs annealed to $200^{\circ} \mathrm{C}$ (Figure S9c) and $250{ }^{\circ} \mathrm{C}$ (Figure S9d). All O 1s peaks were deconvolved into three separate Gaussian-Lorentzian functions, one centred at $529.6 \mathrm{eV}$ (related to $\mathrm{Cu}$ metallic lattice oxide), ${ }^{8}$ one located at $\sim 531.2 \mathrm{eV}$ (which directly relates to Cu defect oxides, ${ }^{8}$ the Pt oxides (Figure S6c) and also charge transfer in Pt group metals) ${ }^{9}$ and one at $\sim 533.4 \mathrm{eV}$ (related to the carbonic oxides). ${ }^{2}$ Interestingly, the concentration of oxide related to the lattice $\mathrm{CuO}$ decreases with deposition of Pt NPs, and further decreases with annealing to $200{ }^{\circ} \mathrm{C}$. This correlates directly to a proportional increase in the function related to increasing $\mathrm{CuO}$ defects, Pt oxide 


\section{Supporting Information}

and charge transfer. Given the low concentration of Pt relatively to $\mathrm{CuO}$, and the subsequent even lower amount of Pt oxides, most of this change is likely due to the $\mathrm{Pt}^{\delta+}$ component in addition to defects created in the CuO lattice created by the decoration of the CuO NWs with the Pt NPs.

Section S4. PeakForce Kelvin probe force microscopy (PF-KPFM) Supplement
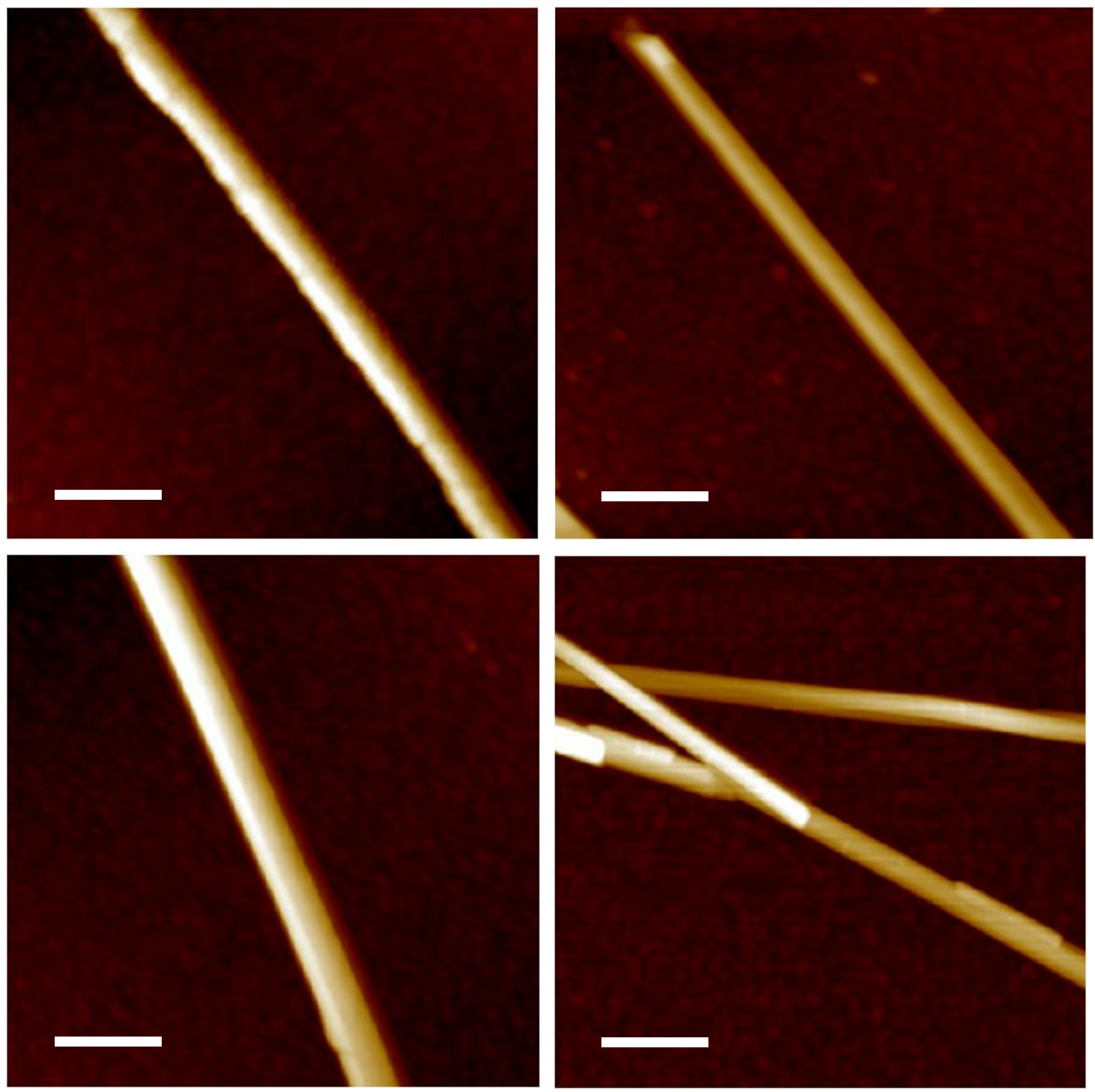

$0 \mathrm{~nm}$

$100 \mathrm{~nm}$

Figure S10. The topographical (height) images corresponding to the surface potential images seen in Figure 4a-d for a) pristine $\mathrm{CuO}$ NW b) as-deposited Pt NPs on CuO NWs and Pt NPs on CuO nanowires annealed at c) $200{ }^{\circ} \mathrm{C}$ and d) $250{ }^{\circ} \mathrm{C}$ in dry synthetic air $\left(80 \% \mathrm{~N}_{2} / 20 @ \mathrm{O}_{2}\right)$ on a Au substrate. The KPMF images were taken with the same magnification and the scale bar indicates $400 \mathrm{~nm}$. 


\section{Supporting Information}
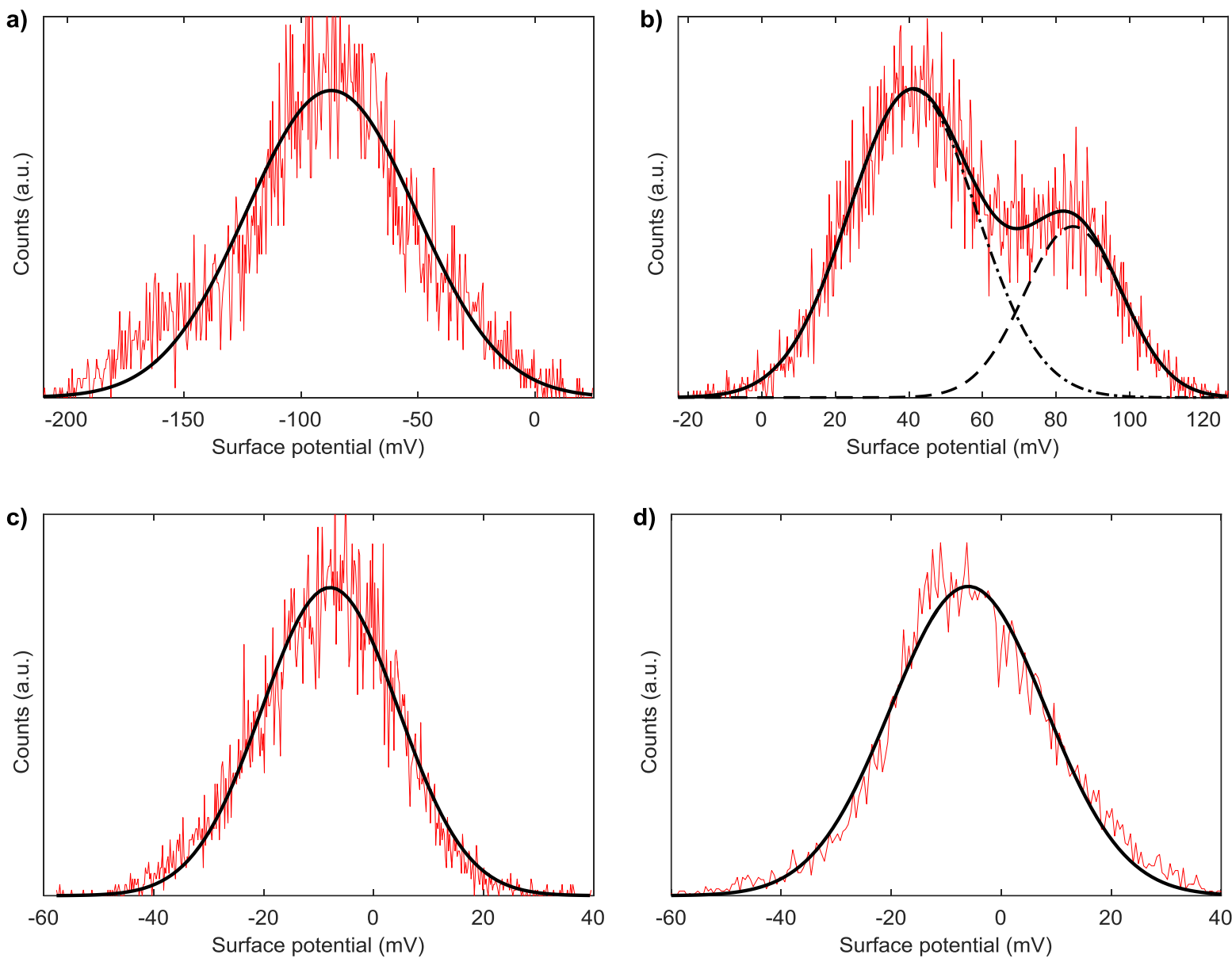

Figure S11. Surface potential distribution profiles fitted with Gaussian distribution of a) a pristine CuO NW, b) as-deposited Pt NPs on CuO NWs, and Pt NPs on CuO nanowires annealed at c) $200{ }^{\circ} \mathrm{C}$ and d) $250{ }^{\circ} \mathrm{C}$ in dry synthetic air. The summary of the mean values together with their standard deviations is displayed in Figure 4e.

The surface potential distribution profile of the annealed Pt NPs decorated CuO NW at $200{ }^{\circ} \mathrm{C}$ was shown in Figure S11b was fitted with two Gaussian distributions. The first one ( $\mu_{1}=41 \mathrm{mV}$ and $\left.\sigma_{1}=12.2\right)$ corresponds to Au substrate (the background of the measurement), while the second one $\left(\mu_{2}=84.5 \mathrm{mV}\right.$ and $\sigma_{2}=9.3$ ) corresponds to the actual Pt NP decorated CuO NWs. 


\section{Supporting Information}

Section S5. Conductometric Measurements of CuO NW based devices and CO Gas Sensing Measuremtns

a)

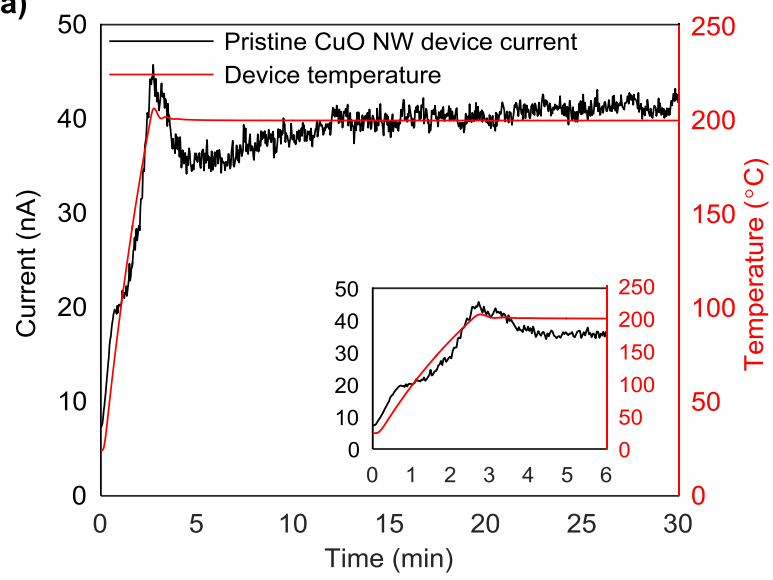

c)

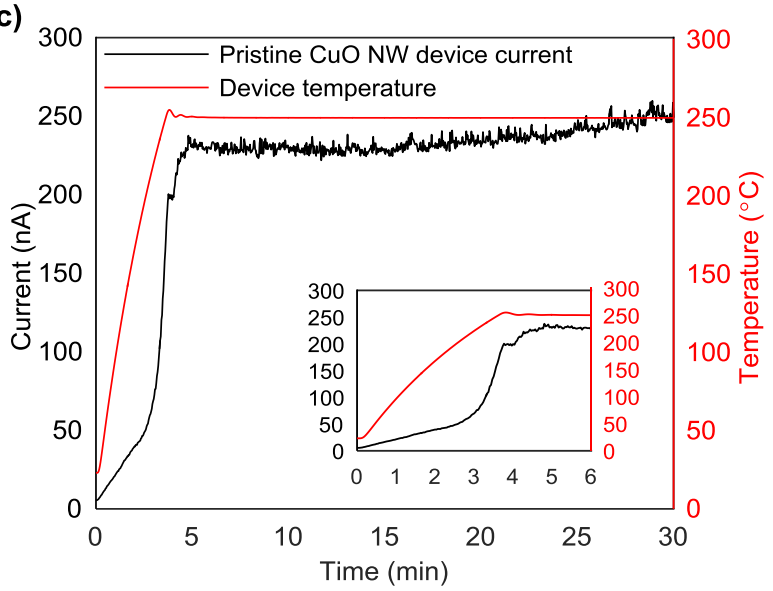

Figure S12. Examples of the current response during the first 30 minutes of the gas sensing measurements (when only synthetic dry air with a ratio of $20 \% 02: 80 \% \mathrm{~N} 2$ was flowing into the system) for the pristine $\mathrm{CuO} \mathrm{NW}$ sensor devices at operating temperature of a) $200^{\circ} \mathrm{C}$ and b) $250^{\circ} \mathrm{C}$. Insets highlight the response of the first 6 minutes of the respective graph. Black lines represent the current measurement, while red lines show the recorded operating temperature. It is important to note that all oscillations in current match with respective oscillations in temperature.

b)

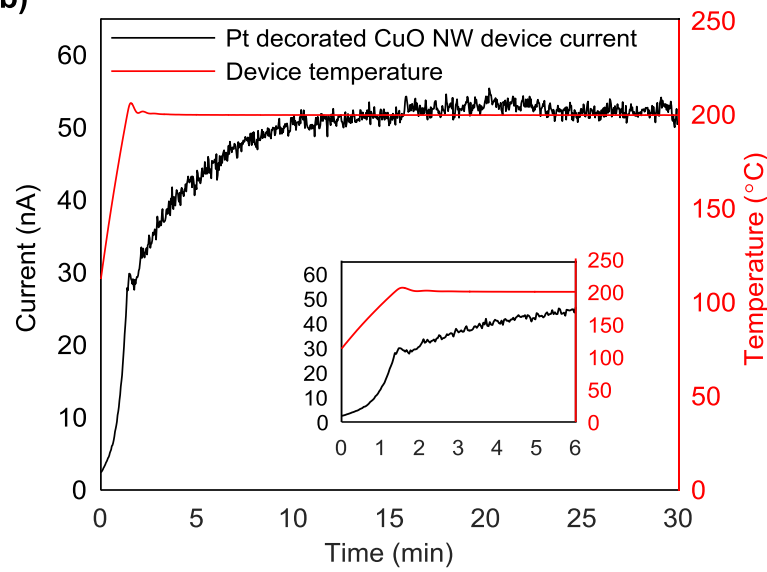

d)

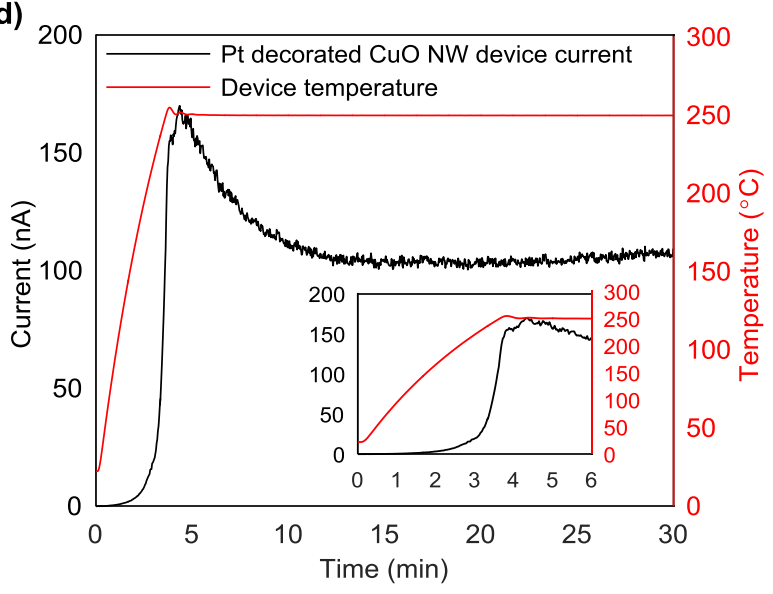

Figure S13. Examples of the current response during the first 30 minutes of the gas sensing measurements (when only synthetic dry air with a ratio of $20 \% \mathrm{O}_{2}: 80 \% \mathrm{~N}_{2}$ was flowing into the system) for the decorated Pt NP-CuO NW sensor devices at operating temperature of a) $200{ }^{\circ} \mathrm{C}$ and b) $250^{\circ} \mathrm{C}$. Insets highlight the response of the first 6 minutes of the respective graph. Black lines represent the current measurement, while red lines show the recorded operating temperature. In this case, the current is showing increases and decreases unrelated to the temperature, indicating an effect by the Pt NPs. This is explained in a) as an increase in oxygen adsorption and spill over increasing the number of holes in the nanowire. In b), after a period of rapid current increase, the current shows a decrease until stability. This is due to oxygen associating with the Pt, and a subsequent competition between Pt NPS and the CuO NWs for the chemisorbed oxygen species. 


\section{Supporting Information}

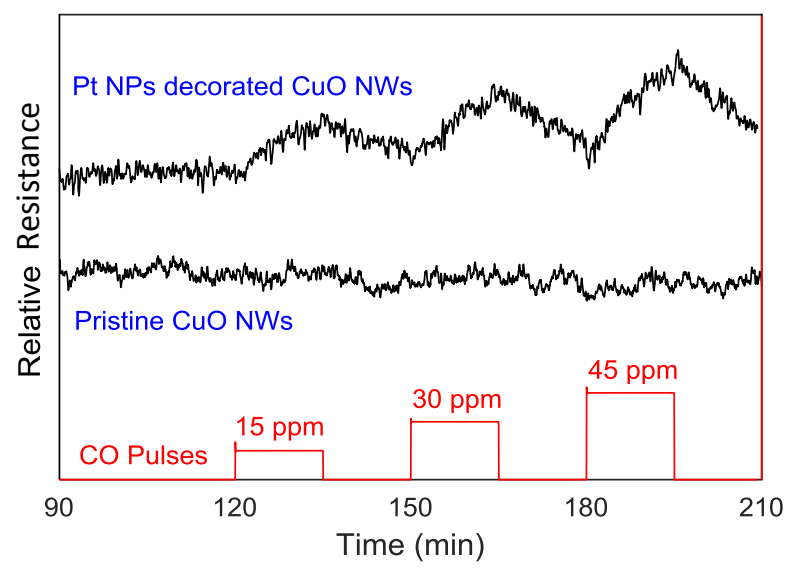

Figure S14. The relative resistance of a Pt decorated CuO NW and a pristine CuO NW devices operated at a temperature of $200^{\circ} \mathrm{C}$ for various concentrations of CO-dry air mixtures.
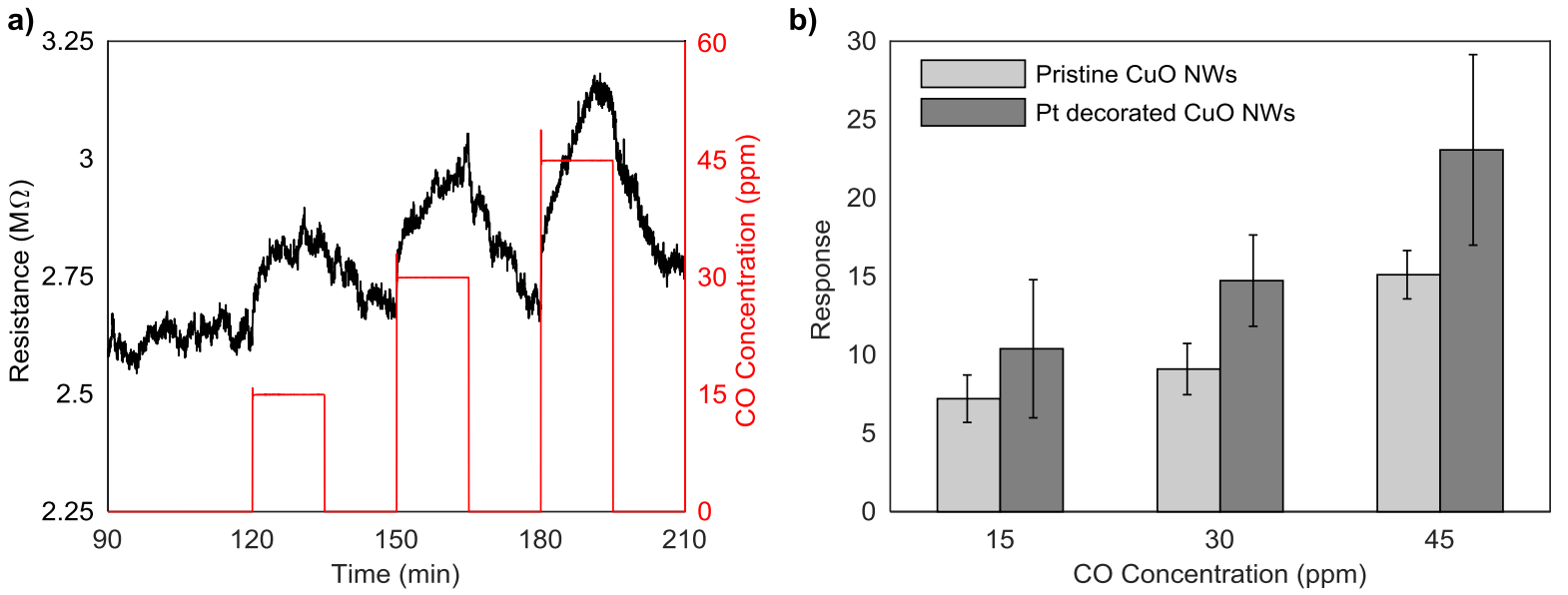

Figure S15. a) The resistance of a pristine CuO NW device operated at a temperature of $250^{\circ} \mathrm{C}$ for different concentrations of CO-dry air mixtures. b) Shows the average response of pristine CuO NW and Pt NP decorated $\mathrm{CuO}$ NW devices (error bars indicate 1 standard deviation) for CO gas. 


\section{Supporting Information}

\section{Section S6. References}

1. Scrocco, M., Satellite structure in the x-ray photoelectron spectra of $\mathrm{CuO} \mathrm{Cu} 2 \mathrm{O}$. Chemical Physics Letters 1979, 63 (1), 52-56.

2. Moulder, J. F.; Stickle, W. F.; Sobol, P. E.; Bomben, K. D., Handbook of X-Ray Photoelectron Epectroscopy: A Reference Book of Standard Spectra for Identification and Interpretation of XPS Data. ULVAC-PHI: Chigasaki, Japan, 1995.

3. Şen, F.; Gökağaç, G., Different Sized Platinum Nanoparticles Supported on Carbon: An XPS Study on These Methanol Oxidation Catalysts. The Journal of Physical Chemistry C 2007, 111 (15), 5715-5720.

4. Dablemont, C.; Lang, P.; Mangeney, C.; Piquemal, J.-Y.; Petkov, V.; Herbst, F.; Viau, G., FTIR and XPS Study of Pt Nanoparticle Functionalization and Interaction with Alumina. Langmuir 2008, 24 (11), 58325841.

5. Kim, J. H.; Woo, H. J.; Kim, C. K.; Yoon, C. S., The catalytic effect of Pt nanoparticles supported on silicon oxide nanowire. Nanotechnology 2009, 20 (23), 235306.

6. Greczynski, G.; Hultman, L., C 1s Peak of Adventitious Carbon Aligns to the Vacuum Level: Dire Consequences for Material's Bonding Assignment by Photoelectron Spectroscopy. Chemphyschem 2017, 18 (12), 1507-1512.

7. Porkovich, A.; Ziadi, Z.; Kumar, P.; Kioseoglou, J.; Jian, N.; Weng, L.; Steinhauer, S.; Vernieres, J.; Grammatikopoulos, P.; Sowwan, M., In Situ Observation of Metal to Metal Oxide Progression: A Study of Charge Transfer Phenomenon at Ru-CuO Interfaces. ACS Nano 2019, 13 (11), 12425-12437.

8. Biesinger, M. C., Advanced analysis of copper X-ray photoelectron spectra. Surface and Interface Analysis 2017, 49 (13), 1325-1334.

9. Wertheim, G. K.; Guggenheim, H. J., Conduction-electron screening in metallic oxides: IrO2. Physical Review B 1980, 22 (10), 4680-4683. 